Article

\title{
Analysis of Possible Noise Reduction Arrangements inside Olive Oil Mills: A Case Study
}

\author{
Simone Pascuzzi ${ }^{+}$(ID) and Francesco Santoro *, + \\ Department of Agricultural and Environmental Science (DiSAAT), University of Bari Aldo Moro, \\ Via Amendola 165/A-70126 Bari, Italy; simone.pascuzzi@uniba.it \\ * Correspondence: francesco.santoro@uniba.it; Tel.: +39-080-544-2474; Fax: +39-080-544-3080 \\ † The Authors equally contributed to the present study.
}

Received: 9 August 2017; Accepted: 11 October 2017; Published: 16 October 2017

\begin{abstract}
Apulia (Southern Italy) is the leading Italian region for the production of olive oil $\left(115 \times 10^{6} \mathrm{~kg}\right.$ of oil/year), and the olive oil chain is really important from a business point of view. Currently, the extraction of olive oil is essentially performed by using a mechanical pressing process (traditional olive oil mills), or by the centrifugation process (modern olive oil mills). The aim of this paper is to evaluate in detail the noise levels within a typical olive oil mill located in the northern part of the Apulia region during olive oil extraction. The feasibility of this study focusing on the assessment of workers' exposure to noise was tested in compliance with the Italian-European Regulations and US standards and criteria. Several measurements of the noise emission produced by each machine belonging to the productive cycle were carried out during olive oil production. The results obtained were then used to evaluate possible improvements to carry out in order to achieve better working conditions. An effective reduction in noise could probably be achieved through a combination of different solutions, which obviously have to be assessed not only from a technical point of view but also an economic one. A significant reduction in noise levels could be achieved by increasing the area of the room allotted to the olive oil extraction cycle by removing all the unnecessary partition walls that might be present.
\end{abstract}

Keywords: olive oil mills; noise pollution; noise reduction; workers' health and safety

\section{Introduction}

The remarkable development in agricultural mechanization in recent years has assured a significant increase in productivity. However, it has led to the requirement for higher attention to detail in all the areas concerning occupational health and safety [1-3], as well as in the exposure of workers' to potentially dangerous physical agents [4-7], activities [8,9] or environments [10]. Amongst these last hazards, of relevant importance is the exposure of workers to noise within plants during food processing [11-13], as is also the case inside olive oil mills during the olive oil extraction process, where noise levels are usually high and should be carefully measured.

Apulia (Southern Italy) is the leading Italian region for the production of olive oil, with a cultivated area of 378,770 hectares, from which about $7.5 \times 10^{8} \mathrm{~kg}$ of olives and $115 \times 10^{6} \mathrm{~kg}$ of olive oil is produced, accounting for $38.6 \%$ of the Italian total [14]. The extraction of olive oil is essentially currently performed by a mechanical pressing process inside traditional olive oil mills, which operate using a discontinuous working cycle, or by the centrifugation process inside modern mills with the use of a continuous cycle of extraction $[15,16]$ (Figure 1). Both these methods require that the olives, washed and with the leaves removed, are previously crushed and reduced to a paste known as "oily juice", from which it is then easier to extract the olive oil. Mechanical crushers connected to mixers perform this step within a continuous working cycle, and the resultant "oily juice" is sent to the 
centrifuge (decanter), which separates it into its three components: pomace; vegetation water, and; olive oil. Instead, inside traditional olive oil mills, stone wheels (roller crusher) crush the olives and the obtained paste is spread on synthetic fiber disks, known as pulp mats, which are stacked and brought to the mechanical press in order to separate the vegetation water and olive oil from the pomace. Finally, in both plant typologies, olive oil and vegetation water are sent to centrifugal separators to extract the olive oil [17].

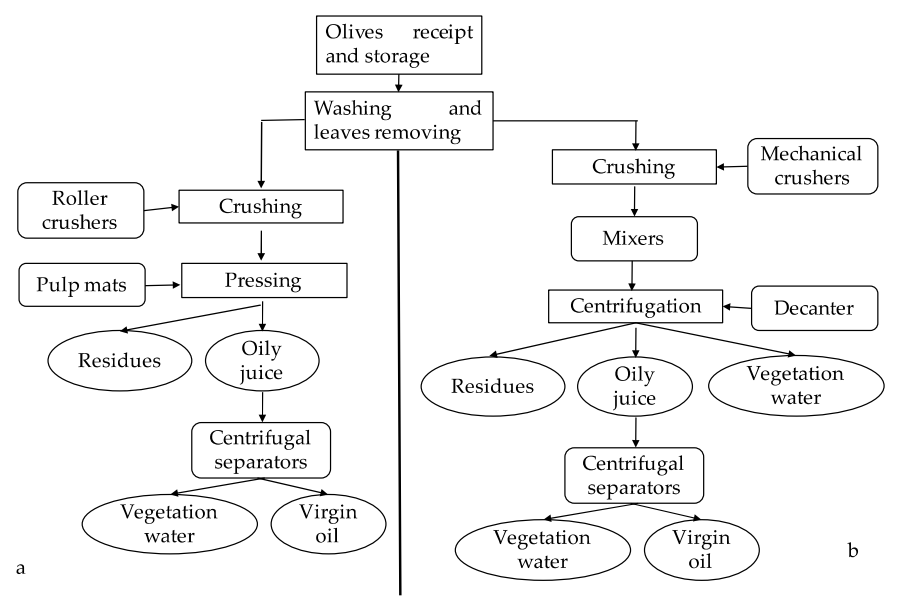

Figure 1. Schematic representation of olive oil extraction lines: (a) "discontinuous" (pressure extraction); (b) "continuous" cycle (extraction by centrifugation).

Olive crushing is a seasonal activity that normally begins at the end of October and finishes at the end of December. During this period, the work is carried out $24 \mathrm{~h}$ a day, seven days a week, and the workers are usually employed with both undefined and fixed-term contracts. The olive oil chain is very important in Apulia from a financial point of view and, even more so in this sector, one of the fundamental objectives of modern mechanized production is safety at work [18]. Regarding this aspect, the manual handling of loads, the use of electricity and noise exposure are the main kinds of risks occurring during working phases. Furthermore, the millers must take the necessary measures to reduce the risk linked to accidental contact with the rotating organs of the working machines, which are potentially accessible not only by the operators of the machine themselves but also by the olive producers who traditionally enter the operating environment of the olive oil mill in order to follow the processing of their own olives [17]. Many Apulian olive oil mills are still located in a single space created in a preexisting or ad hoc structure, even if the evolution of food safety standards requires a physical separation of the operations related to the olives from those related to processing them into olive oil. Therefore, inside the most developed Apulian olive oil mills the leaf removal and the washing phases are carried out in one room, whereas all the processing phases are done in another room [13].

The noise level is usually high during the activity inside the mills, and it is often the case that it is made worse for a wide range of reasons, such as inadequate plant design or improvident labor organization. Therefore, even if the problem of occupational noise exposure for olive oil mill workers is well known, scientific research could still be useful to propose suitable innovative technical solutions consistent with the financial aspects of this typology of factories. Different arrangements and technical solutions are currently used to reduce the noise inside the olive oil mill during the olive oil extraction campaign. These include a change to the layout of the machines, their replacement with newer ones which have lower acoustic impact, or specific actions such as the encapsulation of the noise source inside a box or the inclusion of sound shield or barriers [19]. It is possible to take action to change the characteristics of the acoustics in the working environment through the use of sound-absorbing materials or by means of sound-absorbing panels if the walls produce acoustic reflected waves which, added to the direct acoustic wave coming from the source, produce increased noise. Finally, a different 
organization of the work aimed at reducing the workers' noise exposure time is needed if other technical solutions are not good enough or, as a last line of defense, the use of personal protective equipment such as individual hearing protection devices (muffs, earplugs, headphones) should be considered in order to reduce workers' exposure to noise to below the limits [19].

The regulations in Europe and the US governing the levels of noise permitted in the workplace are someway different, but all of them define the limit for the maximum sound level for a period of eight hours of exposure ( $L_{E X, 8 h}$ or TWA) between 85 and $90 \mathrm{~dB}(\mathrm{~A})$, according to the guidelines of the International Standard ISO 1999:2013 [20]. In the US the standard OSHA (Occupational Safety and Health Administration) 1910.95 establishes values for noise exposure in the workplace and sets for anyone who operates eight hours per day the maximum permissible exposure limit (PEL) to $90 \mathrm{~dB}(\mathrm{~A})$, with an exchange rate of $5 \mathrm{~dB}(\mathrm{~A})$ where an increase of $5 \mathrm{~dB}(\mathrm{~A})$ halves the permitted exposure time [21]. Conversely, the National Institute for Occupational Safety and Health (NIOSH) advises that the equivalent noise level at which a worker is exposed to should be limited to $85 \mathrm{~dB}(\mathrm{~A})$ for an eight-hour day to minimize the risk of hearing damage [22]. In Europe, the risks linked to noise exposure at work are defined in EU Directive 2003/10/EC, which establishes the maximum limit as $87 \mathrm{~dB}(\mathrm{~A})$ for an eight-hour day, even if France, Sweden, Norway and Spain allow $85 \mathrm{~dB}(\mathrm{~A})$ with an exchange rate of $3 \mathrm{~dB}(\mathrm{~A})[23]$.

The Italian Occupational Safety and Health legislation [24], in agreement with the EU Directive [18] and ISO standard [20], establishes that both the worker's exposure time and instantaneous peak exposure must be considered, defining both the peak sound pressure level $\left(L_{p, \text { Cpeak }}\right)$, that is, the highest instantaneous sound pressure weighted through the " $\mathrm{C}$ " ponderation curve, and the daily A-weighted noise exposure level, $L_{E X, 8 h}$, that is, the average value, time-weighted, of all the noise levels at work concerning an eight-hour working day. The Italian law established exposure limit values that are: $L_{E X, 8 h}=87 \mathrm{~dB}$ and $L_{p, \text { Cpeak }}=140 \mathrm{~dB}$ or $200 \mathrm{~Pa}$, respectively [24].

Taking in mind these considerations, the aim of this paper is to deeply evaluate the noise levels inside a typical olive oil mill located in the north of the Apulia region (in Italy) during the olive oil extraction process. This study focuses on the assessment of workers' exposure to noise and was tested in compliance with the Italian legislation, the EU directive and the US standards and criteria. Several measurements concerning the noise emission produced by each machine belonging to the production cycle were carried out during the olive oil production activity. The obtained data and their elaboration were then used to evaluate possible improvements to be made in order to create better working conditions.

\section{Materials and Methods}

\subsection{Tested Olive Oil Mill}

The olive oil mill under consideration, located in the Troia city area (Foggia district, Apulia region, Italy), had a so-called "mixed" or "combined" layout, commonly used in many other olive oil mills found in northern Apulia, as different production chains were integrated, and machines such as the roller crusher pertinent to the traditional discontinuous working cycle operated near to the decanter contrariwise in relation to the modern continuous working one (Figure 2).

In agreement with the extraction process, the obtained paste coming from the roller crushers is first sent to a little mixer, located below the roller crushers, then to a small finisher mechanical crusher and subsequently to the mixer. The paste is then transferred into a decanter and, finally, into the vertical axis centrifugal separators. The production cycle is also able to squeeze olive oil from stoned olives. In this case, the broken drupes coming from the stoner are transferred directly into the mixer and then into the decanter, which operates with the reduced speed of the screw conveyor.

With regards to the structural characteristics of the working environment, the leaf remover and its hopper are located in the olive storage room, built with a concrete floor, tough $4 \mathrm{~m}$ high walls and covered by a galvanized steel sheet (Figure 3). This space is separated from the olive oil extraction 
cycle area by a partition wall, the structural characteristics of which are the following (Figure 3): $4 \mathrm{~m}$ in height, anti-skid flooring, tough plastered walls, tiled up to $1.5 \mathrm{~m}$ above the ground, floor built with prestressed concrete joists and lug bricks, covered in concrete.

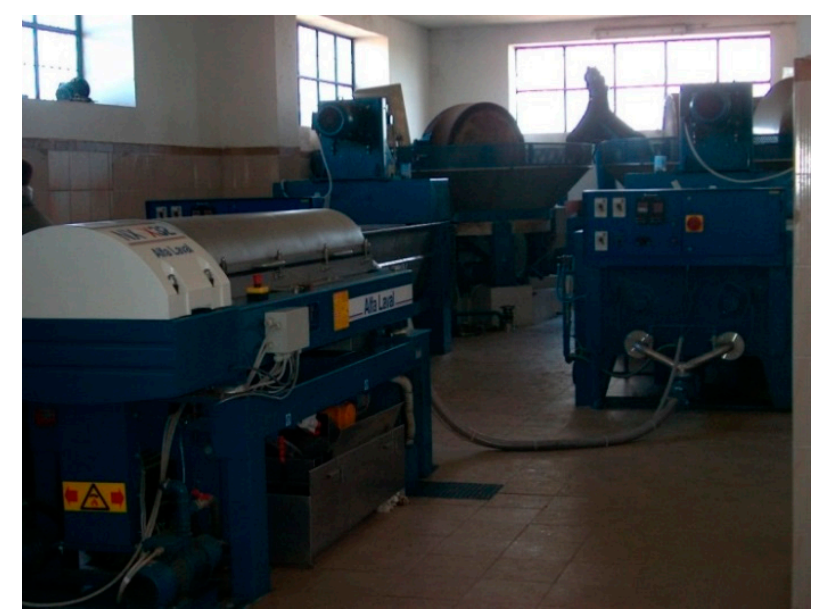

Figure 2. Machines that are pertinent to different working cycles side by side in the tested olive oil mill: the roller crusher (traditional discontinuous working cycle) and the decanter (modern continuous working cycle).

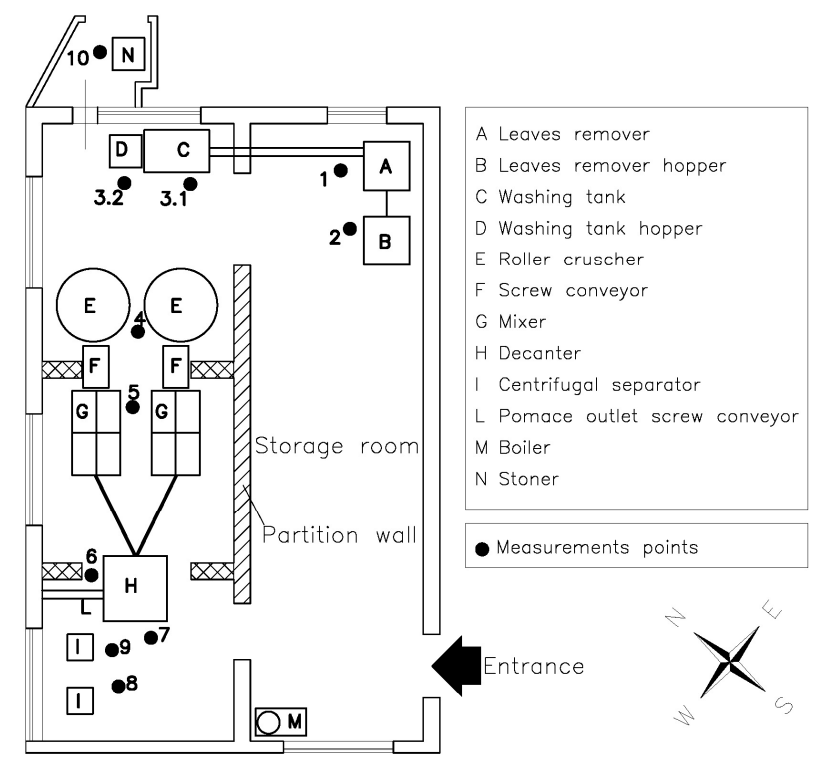

Figure 3. Layout of the tested olive oil mill with measurement points highlighted.

The stoner (Figure 3-N) is placed in a small outdoor room built with tough walls and a galvanized steel cover. Each machine is fastened to the pavement in different ways: (i) the stoner is bolted to the floor with the interposition of vibration reduction mounts; (ii) each roller crusher (Figure 3-E) is fastened to four concrete pillars, which raise the machine off the ground; (iii) the mixers are bolted directly to the floor; (iv) the decanter (Figure 3-H) is supported by a steel frame, which is in turn bolted to the floor with the interposition of vibration reduction mounts; (v) the centrifugal separators (Figure 3-I) are bolted to the floor with the interposition of vibration reduction mounts.

The olive oil mill is managed by a family-owned company and, during the olive oil extraction activities, the mill operates continuously over $24 \mathrm{~h}$; in such conditions, the different workers follow the production, performing rotating shifts of $8 \mathrm{~h}$ each. 


\subsection{Noise Measurements}

The noise measurements were carried out in agreement with the guidelines of the ISO standard in force $[20,25]$. Therefore, since the workers' tasks were limited and well defined, the noise exposure levels during the full activity of the olive oil mill under test were assessed by employing task-based measurements [20].

Taking into account the information gained from the supervisor, the work inside this olive oil mill was performed by all operators in the same way during each shift and so they were regarded as one homogeneous noise exposure group. Furthermore, the workers reported that each of them in rotation spent $1 \mathrm{~h}$ on job planning, briefing and breaks (quiet) with the remaining time equally spent close to the machines. Therefore the average nominal shift was distributed over the following $m=8$ tasks: (1) job planning briefing and breaks (quiet); (2) leaf removal; (3) olive washing; (4) stone removal; (5) crushing; (6) malaxage; (7) settling; (8) centrifugation. An average duration $\overline{T_{m}}=1 \mathrm{~h}$ was considered for each task.

The noise contribution from work planning and breaks was of no importance to the overall noise exposure level. In fact, it was sufficient to carry out some simple noise measurements with the sound level meter, just to ensure that the sound pressure level during these working periods (tasks) had negligible influence. The average value of the actual measurements was $67.8 \mathrm{~dB}$, and then an assessment for such periods was set at $L_{p, A, e q T, 1}=70 \mathrm{~dB}$.

Since the noise contribution from all the machines was highly affected by the location of the workers' ears, it was established to record the sound levels at the measuring points, placed near the following machines (Figure 3): leaf remover (1); leaf remover hopper (2); olive washer (3.1); hopper for the olive washer (3.2); roller crushers (4); mixer (5); decanter (6 and 7); centrifugal separators (8 and 9); stoner (10). The measurements were carried out at $1.5 \mathrm{~m}$ above the floor and at about $1 \mathrm{~m}$ away from the machines, as shown in Figure 3.

The A-weighted equivalent continuous sound pressure level, $L_{p, A, e q T}$ was calculated by the following equation [20]:

$$
L_{p, A, e q T}=10 \lg \left[\frac{\frac{1}{T} \int_{t_{1}}^{t_{2}} p_{A}^{2}(t) \mathrm{dt}}{p_{0}^{2}}\right] \mathrm{dB}
$$

where $p_{a}$ is the A-weighted sound pressure during the stated time interval $T$, starting at $t_{1}$ and ending at $t_{2} ; p_{0}$ is the reference pressure value $(20 \mu \mathrm{Pa})$.

On the other hand, the $C$-weighted peak sound pressure level, $L_{p, \text { Cpeak }}$, was calculated by the following equation [20]:

$$
L_{p, \text { Cpeak }}=10 \lg \frac{p_{\text {Cpeak }}^{2}}{p_{0}^{2}} \mathrm{~dB}
$$

where $p_{0}$ is the reference pressure value $(20 \mu \mathrm{Pa})$.

The measurements of $L_{p, A, e q T}$ and $L_{p, \text { Cpeak }}$ were carried out using a precision sound level meter ACOEM 01dB brand dB4 model, which complies to characteristics imposed by the standards [26,27]: Class 1 sound level meter; Class 0 octave-band and third-octave-band filter; the A weighting scale and slow response $(1 \mathrm{~s})$. The instrument calibration was performed before and after each measurement cycle by means of a calibrator compliant with the standard $[28,29]$. The sound level meter was connected to a G.R.A.S. 46 AC LEMO free-field microphone, having frequency range in the range of $3.15 \mathrm{~Hz}-40 \mathrm{kHz}$ $( \pm 2 \mathrm{~dB})$ and sensitivity of $12.5 \mathrm{mV} / \mathrm{Pa}$ at $250 \mathrm{~Hz}( \pm 1 \mathrm{~dB})$.

Three measurement periods were considered at each of the measuring points and these observations pointed out that the stated time interval $T$ (measurement duration) for noise from all the machines had to be at least equal to $4 \mathrm{~min}$. Therefore, in agreement with the standard, the measurement duration for all the measuring points was set to $5 \mathrm{~min}$ [20]. Furthermore, since the noise from quiet activities was negligible, only some brief samples of noise level were executed during these tasks.

The noise level for each of the eight considered tasks from $l=3$ separate measurements, $L_{p, A, e q T, m}$ has been assessed through the following equation [20]: 


$$
L_{p, A, e q T, m}=10 \cdot \ln \left(\frac{1}{l} \cdot \sum_{1}^{l} 10^{0.1 \cdot L_{p, A e q T, m i}}\right) \mathrm{dB}
$$

where $L_{p, A, e q T, m i}$ is the A-weighted equivalent continuous sound pressure level during a task duration $T_{m} ; i$ the number of task samples $m$ and $l$ the total number of task samples $m$.

The noise level at the "leaf removal" task was calculated considering both the measurements recorded at the measurement points 1 and 2 (Figure 3); in the same way, the noise level at the "olive washing" task was evaluated considering both the measurements recorded at measurement points 3.1 and 3.2, the noise level at the "decanter" task considering both the measurements recorded at measurement points 6 and 7 and, finally, the noise level at the "centrifugal separation" task, considering both the measurements recorded at measurement points 8 and 9 (Figure 3). Practically, $l=6$ was considered for the calculations concerning the "olive washing", "decanter" and "centrifugal separation" tasks.

The contribution from each of the $m$ considered tasks to the daily A-weighted noise exposure level, $L_{E X, 8 h, m}$, was assessed through the following equation [20]:

$$
L_{E X, 8 h, m}=L_{p, A, e q T, m}+10 \lg \left(\frac{\overline{T_{m}}}{T_{0}}\right) \mathrm{dB}
$$

where $\overline{T_{m}}$ is the average duration of the task, that is $1 \mathrm{~h}$, and $T_{0}$ the duration of the nominal shift $\left(T_{0}=8 \mathrm{~h}\right)$.

Finally, the evaluation of the A-weighted noise exposure level, $L_{E X, 8 h}$, from the noise contribution of each of the tasks was executed through the following equation [20]:

$$
L_{E X, 8 h}=10 \lg \left(\sum_{m=1}^{M=8} 10^{0.1 \times L_{E X, 8 h, m}}\right) \mathrm{dB}
$$

where $m$ is the task number and $M$ the total considered number of tasks contributing to the daily noise exposure level $(M=8)$.

In agreement with the US standard and criteria, the noise levels $L_{p, A, q q T, m}$, calculated through Equation (3)for each of the eight considered tasks, were used to compute the total noise dose $D$ over the working day, which is the amount of actual exposure relative to the allowable exposure. $D$ equal to $100 \%$ and above represents exposures that are hazardous. According to both the OSHA standard and the NIOSH recommendations [21,22], $D$ was evaluated through the following equation:

$$
D=100 \cdot\left(\frac{C_{1}}{T_{R D 1}}+\frac{C_{2}}{T_{R D 2}}+\ldots+\frac{C_{8}}{T_{R D 8}}\right)
$$

where $C_{m}$ indicates the total time of exposure at the specific noise level $m$, and $T_{R D m}$ indicates the reference duration for that level.

In agreement with OSHA Regulations 1910.95, which consider $90 \mathrm{~dB}(\mathrm{~A})$ to be the maximum allowable exposure limit with an exchange rate of $5 \mathrm{~dB}(\mathrm{~A})$, the reference duration $T_{R D m(O S H A)}(\mathrm{h})$ was assessed through the following equation [21]:

$$
T_{R D m(O S H A)}=\frac{8}{2^{\left(L_{p, A, e q T, m}-90\right) / 5}}
$$

The eight-hour time-weighted average noise level (TWA) is then computed from the daily dose $D$, by means of the following formula [21]:

$$
\operatorname{TWA}_{(\mathrm{OSHA})}=16.61 \lg \left(\frac{D}{100}\right)+90 \mathrm{~dB}
$$

Conversely, according to the NIOSH recommendations, which consider $85 \mathrm{~dB}(\mathrm{~A})$ the maximum permissible exposure limit with an exchange rate of $3 \mathrm{~dB}(\mathrm{~A})$, the reference duration $T_{R D m(N I O S H)}(\mathrm{h})$ was assessed through the following equation [22]: 


$$
T_{R D m(N I O S H)}=\frac{8}{2^{\left(L_{p, A, e q T, m}-85\right) / 3}}
$$

In this case, the TWA was evaluated by means of the following formula [22]:

$$
T W A_{(\mathrm{NIOSH})}=10.0 \lg \left(\frac{D}{100}\right)+85 \mathrm{~dB}
$$

\section{Results and Discussion}

Figure 4 shows the A-weighted equivalent continuous sound pressure levels $L_{p, A, e q T, m}$ and the C-weighted peak sound pressure levels $L_{p, \text { Cpeak }}$ concerning the measurements carried out at each considered measured point. The range of the three measured values of $L_{p, A, e q T, m}$ never exceeded $3 \mathrm{~dB}$, and then no additional measurements were made [19]. Conversely, the graph highlights that $L_{p, C p e a k}$ overcame the limit of $140 \mathrm{~dB}$ at no measuring point, and the lowest peak ( $90.1 \mathrm{~dB}$ ) took place near the washing tank, whereas the highest one $(102.3 \mathrm{~dB})$ is near the mixer.

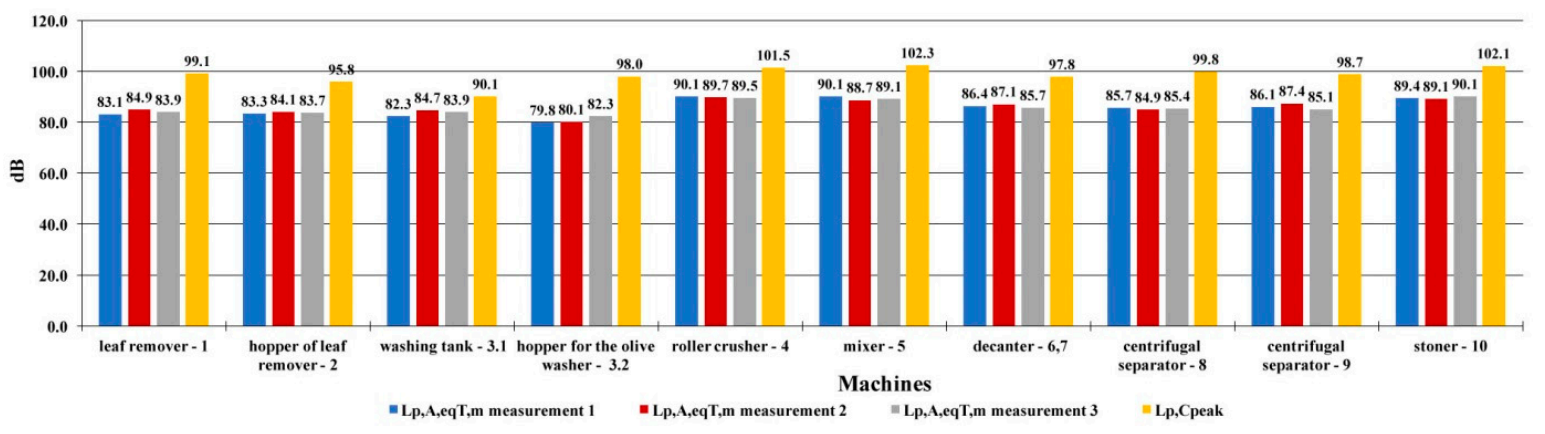

Figure 4. A-weighted equivalent continuous sound pressure levels $\left(L_{\mathrm{p}, \mathrm{A}, \mathrm{eqT}, \mathrm{m}}\right)$ and $\mathrm{C}$-weighted peak sound pressure levels $\left(L_{\mathrm{p}, \mathrm{Cpeak}}\right)$ registered at the considered measuring point.

The noise level for each of the eight considered tasks $L_{p, A, e q T, m}$ are reported in Figure 5. They were calculated using Equation (3), taking into account the data pointed out in Figure 4. The chart highlights that the noisiest machines, that is, the ones with $L_{p, A, e q T, m}$ higher than $87 \mathrm{~dB}$, which were the roller crushers, the mixer and the stoner, even if the last one is located in a separate room and isolated from the other machines directly involved in the production cycle (Figure 3). The results highlight the fact that workers compelled to always operate near these machines would be subjected to a daily noise exposure greater than the limit; luckily, the operation connected to these machines does not require the constant presence of employees.

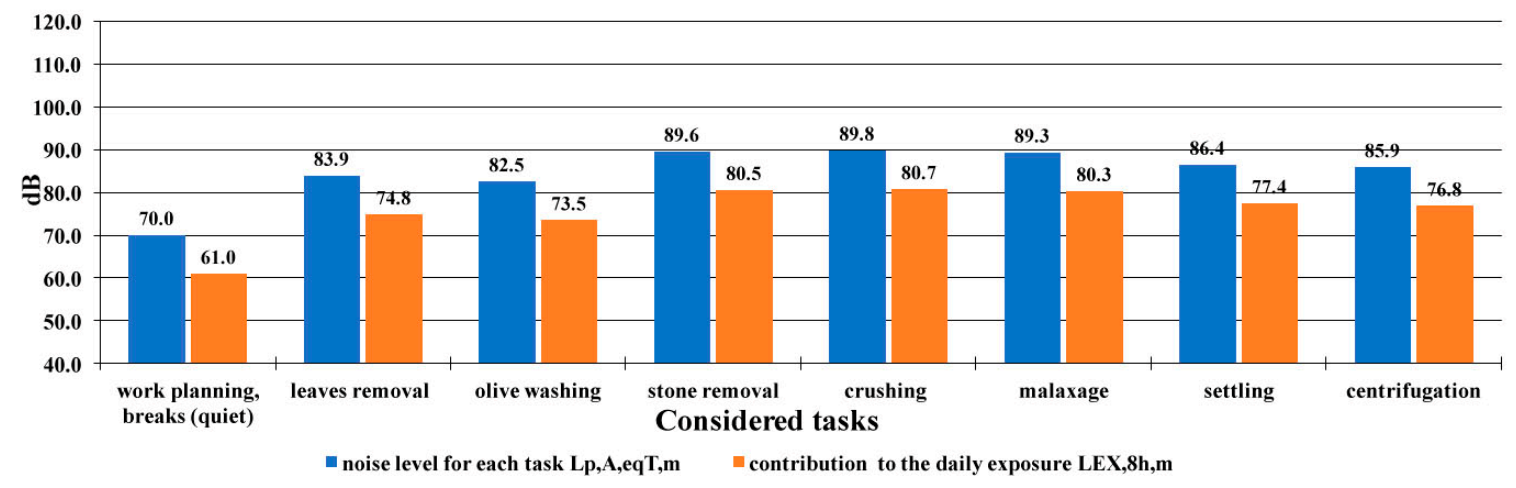

Figure 5. Noise level for $L_{p, A, e q T, m}$ and contribution to the daily A-weighted noise exposure level $\mathrm{L}_{\mathrm{EX}, 8 \mathrm{~h}, \mathrm{~m}}$ concerning the considered tasks. 
Figure 5 also reports the contribution to the daily A-weighted noise exposure level, $\mathrm{L}_{E X, 8 h, m \text {, }}$ calculated for each activity according to Equation (4). As previously stated, the average task duration of all the considered activity was the same $(1 \mathrm{~h})$, and so the bar chart concerning the $L_{E X, 8 h, m}$ has the same shape and is only proportionally reduced with reference to the one that is relevant to $L_{p, A, e q T, m}$. In addition, this graph points out that none of the activities contribute to overcoming the limit of $87 \mathrm{~dB}$.

The reference durations $T_{R D m}(\mathrm{~h})$, calculated according to both the OSHA Regulations 1910.95 and the NIOSH recommendations, respectively, through Equations (7) and (9), are reported in Figure 6. The chart points out the significant difference between the two approaches, mainly with reference to the noisiest machines. For example, the NIOSH recommends staying no closer to the roller crusher than $T_{R D m}=2.7 \mathrm{~h}$, whereas by the OSHA standard $8.3 \mathrm{~h}$ are permitted $(+207 \%)$, so for the stoner where $T_{R D m}$ is $2.8 \mathrm{~h}$ by NIOSH, and $T_{R D m}$ is $8.5 \mathrm{~h}$ by OSHA (+204\%). The average task duration of all the considered activity $\left(\overline{T_{m}}=1 \mathrm{~h}\right)$ was in any case less than the computed values reported in Figure 6.

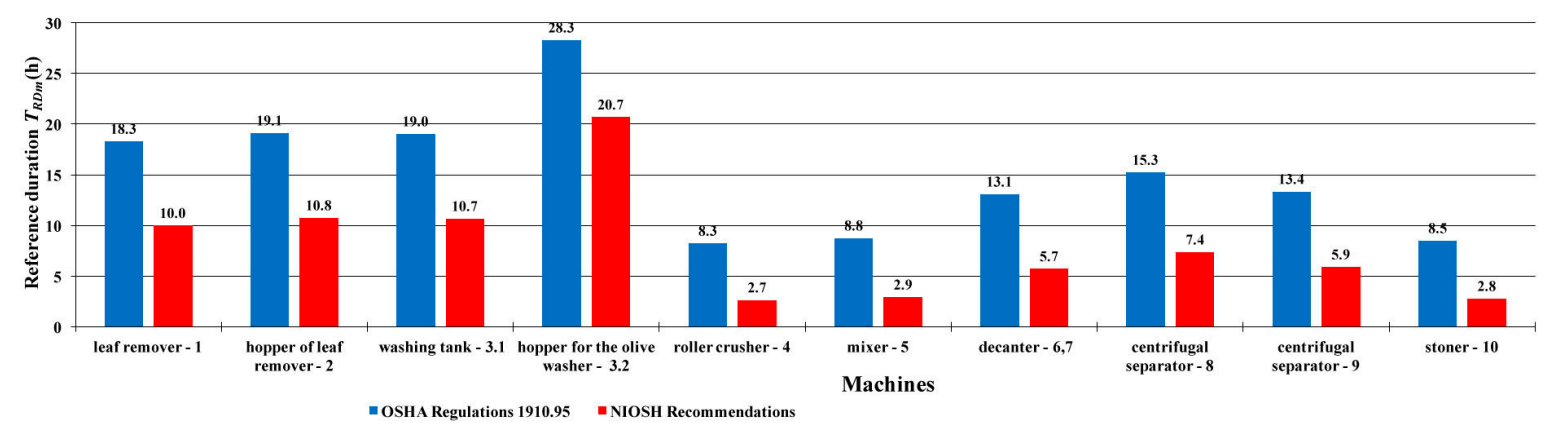

Figure 6. Reference durations $T_{R D m}(\mathrm{~h})$, calculated according to both the OSHA Regulations 1910.95 and the National Institute for Occupational Safety and Health (NIOSH) recommendations.

Table 1 reports the calculated values concerning the total daily dose $D$ and the eight-hour time-weighted average noise level TWA with the corresponding limits according to both European and US standards. The dose $D$, not considered by the ISO standard, is very different if calculated according to OSHA 1910.95 or NIOSH criteria and the computed value is lower than the admissible one if evaluated through the OSHA standard. Conversely, $D$ is much greater than the allowed one in compliance with the NIOSH criteria; moreover, the corresponding TWA, assessed through Equation (10), is obviously higher than the threshold value (Table 1). For the operators whose noise exposures equal or exceed $85 \mathrm{~dB}(\mathrm{~A})$, NIOSH advises a hearing loss articulated prevention program, which contains exposure evaluation, engineering and administrative controls, suitable employment of hearing protectors, audiometric evaluation, education and motivation and recordkeeping [21].

Table 1. Average duration of the $m$ considered tasks during the nominal shift. Time-weighted average noise level (TWA).

\begin{tabular}{|c|c|c|c|}
\hline & \multirow[t]{2}{*}{$\begin{array}{c}\text { Daily Noise Dose } \\
\text { D\% }\end{array}$} & \multicolumn{2}{|c|}{$\begin{array}{c}8 \mathrm{~h} \text { Work Shift Noise Exposure Level } \\
\mathrm{L}_{E X, 8 h} \text {-TWA dB(A) }\end{array}$} \\
\hline & & Computed & Limit \\
\hline OSHA 1910.95 & 76.4 & 88.1 & 90 \\
\hline NIOSH criteria & 188.9 & 87.8 & 85 \\
\hline Italian Regulations & / & 86.9 & 87 \\
\hline
\end{tabular}

The daily A-weighted noise exposure level, calculated through Equation (5) in agreement with the EU Directive $L_{E X, 8 h, m}=86.9 \mathrm{~dB}$, is lower than the corresponding limit, even if this value is higher than the exposure action value, which is $85 \mathrm{~dB}$, so actions aimed at reducing the sound level and protecting workers' well-being have to be undertaken. 
Regardless of the values pertinent to the different technical or law-making approaches, the results obtained highlight the considerable level of noise in the places where operations to process olives into olive oil are carried out, thereby emphasizing the necessity to analyze the feasibility of every possible technical solution aimed at minimizing the risk of damaging hearing and other possible types of harm such as cardiovascular diseases, fatigue, inability to concentrate and reduced motivation [30-32], and diastolic blood pressure increase [33-35].

According to the structural characteristics of the tested olive mill, together with the results of the noise level measurements, it is possible to propose some feasible arrangements aimed at reducing the noise emissions during the olive oil extraction cycle. It is useful to point out that an effective improved result in terms of noise reduction could probably be achieved through a combination of different solutions, which obviously have to be assessed not only from a technical point of view but also from an economic one. Inside the tested olive oil mill, the whole extraction cycle essentially takes places in one room, which is too small considering the number and dimensions of the machines used. There is very little free space around the machines so, in addition to hampering workers' movements, there is a higher noise level in the environment due to the occurrence of acoustic reflections. Furthermore, the structural characteristics and the dimension of the olive oil mill, together with the layout of the machines, do not allow their encapsulation, first because this solution would further decrease workers' available space. Finally, the noise level is also due to the overlap of the direct sound fields generated by machines with the indirect ones caused by the multiple reflections on the walls, so the use of noise barriers, even if placed close to the sources, may not be useful in order to obtain the expected result of reducing noise levels. The key solution in order to increase the workers' safety and their operative conditions is to eliminate the partition wall that currently separates the storage room from the room used for the olive oil extraction cycle itself (Figure 3). This new structural configuration will leave more space available to accommodate machines that will then be arranged suitably from one another. Further reductions in sound emissions can be achieved by encapsulating the noisiest machines such as the roller crusher, the mixer and the stoner. The acoustical features of the new working environment can also be varied by reducing the reflected noise waves emitted by the machines and walls by way of sound-absorbing materials or sound-absorbing panels. Personal protective equipment ultimately could be taken into account in the event that after the executed changes, noise issues still bother the workers. In any case, the plant design solutions in conjunction with suitable job organization will provide functional opportunities for operators to do their work effectively and efficiently without undue distraction or threat.

\section{Conclusions}

The noise levels inside a typical olive oil mill located in Southern Italy were analyzed during the operations for processing olives into olive oil. The workers' exposure to noise was assessed in compliance with the Italian-European and US Regulations. The obtained results reveal a high level of noisiness inside the working environment, and the sound measured values were very close to the corresponding threshold values covered by both the Italian-European Regulations and the US Standard, whereas they were well over the limits recommended by the US National Institute for Occupational Safety and Health (NIOSH). The executed measurements also highlight the necessity to mitigate the noise levels within the olive oil mills through solutions, doubtless cumbersome and involving technical, economic and organizational aspects. In reality, the achievement of the target could be reached by way of the synergic effect of more combined actions and consequently an integrated analysis of the problem is required. Referring to the specific tested olive oil mill, the available space where the olive oil extraction cycle takes place is not actually suitable to host all the machines used and, in addition to hampering workers' movements, widespread noise due to multiple reflections along the walls occurs. A significant reduction in the noise levels could be achieved by increasing the size of the room used for the olive oil extraction cycle by removing the partition wall that separates this room from the one next to it, which is devoted to the stockpile of the olives. It is useful to note that every 
technical analysis of the problem should not be kept apart from an accurate economic assessment, since the seasonal nature of work inside the olive oil mills, and the small earnings due to the high competitiveness, could be the reason for the inability to put into place any of the technical evaluated measures. These are measures which, although effective, could be too expensive from a financial point of view, even though it should be considered that the economic costs involved in the improvement of workers' safety and operative conditions are doubtless smaller than those (social as well as economic) linked to accidents at work: investing in prevention is always a good strategy.

Author Contributions: Both Authors conceived, designed and performed the experiments, did data collection, analysis and interpretation, wrote, revised and proofread the paper equally.

Conflicts of Interest: The authors declare no conflict of interest.

\section{Abbreviations}

TWA Time Weighted Average (Noise Levels)

PEL Permissible Exposure Limit

$L_{p, A, e q T} \quad$ A-weighted equivalent continuous sound pressure level

$L_{p, A, e q T, m} \quad$ A-weighted equivalent continuous sound pressure level during a task

$L_{p, \text { Cpeak }} \quad$ Peak sound pressure level

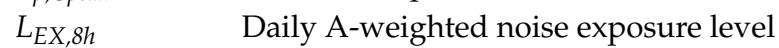

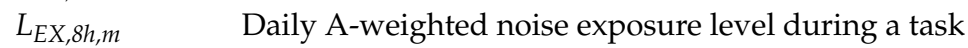

$D \quad$ Total daily noise dose

$T_{R D m(O S H A)} \quad$ Reference duration time according OSHA

TWA (OSHA) Time Weighted Average (Noise Levels) according OSHA

$T_{R D m(N I O S H)} \quad$ Reference duration time according NIOSH

$T W A_{(N I O S H)} \quad$ Time Weighted Average (Noise Levels) according NIOSH

\section{References}

1. Pascuzzi, S. A multibody approach applied to the study of driver injuries due to a narrow-track wheeled tractor rollover. J. Agric. Eng. 2015, 46, 105-114. [CrossRef]

2. Pascuzzi, S. The effects of the forward speed and air volume of an air-assisted sprayer on spray deposition in "tendone" trained vineyards. J. Agric. Eng. 2013, 3, 125-132. [CrossRef]

3. Baldoin, C.; Balsari, P.; Cerruto, E.; Pascuzzi, S.; Raffaelli, M. Improvement in pesticide application on greenhouse crops: Results of a survey about greenhouse structures in Italy. Acta Hortic. 2008, 801, 609-614. [CrossRef]

4. Pascuzzi, S.; Santoro, F. Evaluation of farmers' OSH hazards in operation nearby mobile telephone radio base stations. In Proceedings of the 16th International Scientific Conference on Engineering for rural development, Jelgava, Latvia, 24-26 May 2017; Latvia University of Agriculture-Faculty of Engineering: Jelgava, Latvia, 2017; pp. 748-755. [CrossRef]

5. Pascuzzi, S.; Santoro, F. Exposure of farm workers to electromagnetic radiation from cellular network radio base stations situated on rural agricultural land. Int. J. Occup. Saf. Ergon. 2015, 21, 351-358. [CrossRef] [PubMed]

6. Pascuzzi, S.; Blanco, I.; Anifantis, A.S.; Scarascia Mugnozza, G. Hazard assessment and technical actions due to the production of pressured hydrogen within a pilot photovoltaic-electrolyzer-fuel cell power system for agricultural equipment. J. Agric. Eng. 2016, 47, 89-93. [CrossRef]

7. Marucci, A.; Pagniello, B.; Monarca, D.; Colantoni, A.; Biondi, P. Heat stress suffered by workers employed in vegetable grafting in greenhouses. J. Food Agric. Environ. 2012, 10, 1117-1121.

8. Boubaker, K.; Colantoni, A.; Allegrini, E.; Longo, L.; Di Giacinto, S.; Monarca, D.; Cecchini, M. A model for musculoskeletal disorder-related fatigue in upper limb manipulation during industrial vegetables sorting. Int. J. Ind. Ergon. 2014, 44, 601-605. [CrossRef]

9. Colantoni, A.; Marucci, A.; Monarca, D.; Pagniello, B.; Cecchini, M.; Bedini, R. The risk of musculoskeletal disorders due to repetitive movements of upper limbs for workers employed to vegetable grafting. J. Food Agric. Environ. 2012, 103, 14-18. 
10. Di Giacinto, S.; Colantoni, A.; Cecchini, M.; Monarca, D.; Moscetti, R.; Massantini, R. Dairy production in restricted environment and safety for the workers. Ind. Aliment. 2012, 530, 5-12.

11. Manetto, G.; Cerruto, E.; Pascuzzi, S.; Santoro, F. Improvements in citrus packing lines to reduce the mechanical damage to fruit. Chem. Eng. Trans. 2017, 58, 391-396. [CrossRef]

12. Bianchi, B.; Tamborrino, A.; Santoro, F. Assessment of the energy and separation efficiency of the decanter centrifuge with regulation capability of oil water ring in the industrial process line using a continuous method. J. Agric. Eng. 2013, 44, 278-282. [CrossRef]

13. Cecchini, M.; Contini, M.; Massantini, R.; Monarca, D.; Moscetti, R. Effects of controlled atmospheres and low temperature on storability of chestnuts manually and mechanically harvested. Postharvest Biol. Technol. 2011, 61, 131-136. [CrossRef]

14. Italian National Institute of Statistics (ISTAT). Area (Hectares) and Production (Quintals) of Olives 2016. Available online: http:/ /agri.istat.it/sag_is_pdwout/jsp/NewDownload.jsp?id=15A \T1 $\backslash$ textbar\{\}21A \T1 \ textbar\{\}32A\&anid=2016 (accessed on 5 July 2017).

15. Leone, A.; Romaniello, R.; Tamborrino, A.; Xu, X.Q.; Juliano, P. Microwave and megasonics combined technology for a continuous olive oil process with enhanced extractability. Innov. Food Sci. Emerg. Technol. 2017, 42, 56-63. [CrossRef]

16. Vivaldi, G.A.; Strippoli, G.; Pascuzzi, S.; Stellacci, A.M.; Camposeo, S. Olive genotypes cultivated in an adult high-density orchard respond differently to canopy restraining by mechanical and manual pruning. Sci. Hortic. 2015, 192, 391-399. [CrossRef]

17. Clodoveo, M.L.; Camposeo, S.; de Gennaro, B.; Pascuzzi, S.; Roselli, L. In the ancient world virgin olive oil has been called "liquid gold" by Homer and the "great healer" by Hippocrates. Why is this mythic image forgotten? Food Res. Int. 2014, 62, 1062-1068. [CrossRef]

18. Manetto, G.; Cerruto, E. Vibration risk evaluation in hand-held harvesters for olives. J. Agric. Eng. 2013, 44, 705-709. [CrossRef]

19. Cirillo, E. Applied Acoustics; McGraw-Hill: Trento, Italy, 1997; 216p. (In Italian)

20. International Organization for Standardization. Acoustics-Estimation of Noise-Induced Hearing Loss; ISO 1999:2013; International Organization for Standardization: Geneva, Switzerland, 2013.

21. U.S. Occupational Safety and Health Administration. OSHA 1910.95. Available online: https://www.osha.gov/ pls /oshaweb / owadisp.show_document?p_table=standards\&p_id=9735 (accessed on 22 September 2017).

22. U.S. Department of Health and Human Services. Department of Health and Human Services. Revised Criteria 1998. Available online: https:/ /www.cdc.gov/niosh/docs/98-126/pdfs/98-126.pdf (accessed on 23 August 2017).

23. EU. Directive 2003/10/EC on the Minimum Health and Safety Requirements Regarding the Exposure of Workers to the Risks Arising from Physical Agents (Noise); European Parliament and the Council: Brussels, Belgium, 2003.

24. Official Gazette of the Italian Republic. Safety and Health in Workplaces Act of 2008; Italian Law Decree No. 81 (9 April 2008); Official Gazette of the Italian Republic: Rome, Italy, 2008. (In Italian)

25. International Organization for Standardization (ISO). Acoustics-Determination of Occupational Noise Exposure-Engineering Method; ISO 9612:2009; International Organization for Standardization: Geneva, Switzerland, 2009.

26. UNI. Acoustics-Determination of Occupational Noise Exposure; UNI 9432:2011; Italian Organization for Standardization: Milan, Italy, 2011. (In Italian)

27. International Electrotechnical Commission (IEC). Electroacoustic_Sound Level Meters_Part 1: Specification; IEC 61672-1:2013; International Electrotechnical Commission: Geneva, Switzerland, 2013.

28. International Electrotechnical Commission (IEC). Electroacoustic-Octave-Band and Fractional-Octave-Band Filters_Part 1: Specification; IEC 61260-1:2014; International Electrotechnical Commission: Geneva, Switzerland, 2014.

29. International Electrotechnical Commission (IEC). Electroacoustic-Sound Calibration; IEC 60942:2003; International Electrotechnical Commission: Geneva, Switzerland, 2003.

30. Lercher, P.; Hortnagl, J.; Kofler, W. Work Noise Annoyance and Blood Pressure: Combined Effects with Stressful Working Conditions. Int. Arch. Occup. Environ. Health 1993, 65, 23-28. [CrossRef] [PubMed]

31. Loewen, L.; Suedfeld, P. Cognitive and arousal effects of masking office noise. Environ. Behav. 1992, 24, 381-395. [CrossRef] 
32. Evans, G.W. Environmental stress and health. In Handbook of Health Psychology; Baum, A., Revenson, T., Singer, J., Eds.; Wiley: New York, NY, USA, 2001; Volume 1, pp. 571-610.

33. Melamed, S.; Fried, Y.; Froom, P. The interactive effect of chronic exposure to noise and job. J. Occup. Health Psychol. 2001, 6, 182-195. [CrossRef] [PubMed]

34. Van Kempen, E.E.; Kruize, H.; Boshuizen, H.C.; Ameling, C.B.; Staatsen, B.A.M.; de Hollander, A.E.M. The association between noise exposure and blood pressure and ischemic heart disease: A meta-analysis. Environ. Health Perspect. 2002, 110, 307-317. [CrossRef] [PubMed]

35. Willich, S.N.; Wegscheider, K.; Stallmann, M.; Keil, T. Noise burden and the risk of myocardial infarction. Eur. Heart J. 2006, 27, 276-282. [CrossRef] [PubMed]

(C) 2017 by the authors. Licensee MDPI, Basel, Switzerland. This article is an open access article distributed under the terms and conditions of the Creative Commons Attribution (CC BY) license (http://creativecommons.org/licenses/by/4.0/). 